\title{
Hydrogen Adsorption of Acid-treated Multi-walled Carbon Nanotubes at Low Temperature
}

\author{
Seul-Yi Lee and Soo-Jin Park* \\ Department of Chemistry, Inha University, Incheon 402-751, Korea. "E-mail: sjpark@inha.ac.kr \\ Received March 26, 2009, Accepted March 25, 2010
}

\begin{abstract}
Surface functionalization of multi-walled carbon nanotubes (MWNTs) was carried out by means of acid treatment. The presence of oxygen functional groups on the surface of acid-treated MWNTs was confirmed with the aid of Fourier transform infrared spectroscopy and X-ray spectroscopy. In addition, carboxylic groups generally formed on the surface of acid-treated MWNTs, and the dispersion was increased by the duration of the acid treatment. The zeta-potential indicated the surface charge transfer and the dispersion of MWMTs. Morphological characteristics of acid-treated MWNTs were also observed using a transmission electron microscopy, X-ray diffraction, and Raman analysis, which was revealed the significantly unchanged morphologies of MWNTs by acid treatment. The hydrogen adsorption capacity of the MWNTs was evaluated by means of adsorption isotherms at $77 \mathrm{~K} / 1 \mathrm{~atm}$. The hydrogen storage capacity was dependent upon the acid treatment conditions and the formation of oxygen functional groups on the MWNT surfaces. The latter have an important effect on the hydrogen storage capacity.
\end{abstract}

Key Words: MWNT, Acid treatment, Hydrogen adsorption, Oxygen-functional groups

\section{Introduction}

Hydrogen is one of the most promising alternative fuels for the foreseeable future. However, it can only be used in a liquid phase in critical surroundings because of its extremely low temperature (of about $20.3 \mathrm{~K}$ ). For this reason, hydrogen is difficult to control as an energy carrier and hydrogen storage is critical for safe and efficient application. Researchers are consequently endeavoring to overcome this disadvantage by increasing the efficiency of hydrogen storage materials. Some technologies that store hydrogen as a liquid, in gas phases, or in hydrogen storage materials have been actively investigated. ${ }^{1,2}$ In particular, carbon nanomaterials with a low mass density, a high specific surface, and a surface defect have looked forward having hydrogen storage capacity. ${ }^{3,4}$

Carbon materials offer many advantages for hydrogen storage, such as low mass density, good surface functional groups, and high surface area. Carbon nanotubes (CNTs) in particular are known to display significant catalytic activity by means of $\pi$ and $\sigma$ bonds; the latter is associated with carbon in graphitic sheets. Five- or seven- carbon rings of CNTs may be compounded for a hexagonal network of graphitic sheets. These carbon rings have a lower level of stability than a set of six rings and are susceptible to the introduction of functional groups in a chemical process. ${ }^{5-7}$ Furthermore; nano-sized CNTs with a high aspect ratio provide abundant additional interface sites where most of the important chemical reactions occur. However, CNTs tend to agglomerate in a soluble, which greatly weakens their mechanical strength, electrical performance, and so on. A process of modifying the surface of the CNTs is essential if these problems are to be overcome. In addition, the chemical reactivity of the graphitic network can be enhanced by the oxidation of CNTs. The oxidation can occur as a result of a gas phase treatment or wet chemical methods, such as photo-oxidation and oxygenplasma. Through these treatments, crude CNTs can be effectively purified and the introduced oxygen functional groups, mainly carboxyl and hydroxyl, can emerge to decorate the CNT surfaces. The oxygen functional groups on MWNTs are important for improving the solubility of polar media and the storage capacity of hydrogen. ${ }^{8-10}$

The aim of present work is to investigate the dispersion behaviors and characteristics of functional groups on CNT surfaces modified by acid treatment. At the same time, the relation between the hydrogen adsorption properties and functional groups of MWNT surfaces is studied in terms of the acid treatment.

\section{Experimental Section}

Sample preparation. CNT crude materials (MWNTs, purity: $90 \%$ ) produced by chemical vapor deposition were purchased from the Nano Solution Co. Ltd (Korea). The MWNTs had a diameter of 10 to $30 \mathrm{~nm}$ and a length of 20 to $50 \mu \mathrm{m}$. The MWNTs were chemically purified in $5 \mathrm{M}$ of nitric acid for $2 \mathrm{~h}$ at room temperature. They were filtered and thoroughly washed with distilled water several times and then dried in a vacuum oven for $24 \mathrm{~h}$ at $60{ }^{\circ} \mathrm{C}$. The purified MWNTs were immersed in $2 \mathrm{M}$ of phosphoric acid: treating for $2 \mathrm{~h}$ (T-2), for $5 \mathrm{~h}$ (T-5), and for $10 \mathrm{~h}(\mathrm{~T}-10)$. The remaining untreated ones are called as-received MWNTs.

Characterization. Fourier transform infrared spectroscopy (FT-IR) was used to investigate the functional groups that formed as a result of the acid-treated MWNTs. The spectrometer was from Jasco International Co., Ltd. (Model FT/IR-4200).

For the X-ray spectroscopy (XPS), an achromatic $\mathrm{MgK} \alpha$ $\mathrm{X}$-ray source was used at $150 \mathrm{~W}$. Survey scans at about $\mathrm{C}_{1 \mathrm{~S}}$ were collected from a range of 270 to $300 \mathrm{eV}$ with a pass energy equal to $20 \mathrm{eV}$. The vacuum system pressure was maintained at approximately $10^{-9}$ torr during all XPS experiments. A nonlinear least squares curve fitting program (Peak-Fit version 4) with a Gaussian-Lorentzian mix function and Shirley back- 
ground subtraction was used to deconvolve the XPS subpeaks.

The zeta-potential measurements were performed with a zetapotential analyzer from Otsuka Electronics Co., Ltd. For dispersion, the samples were sonicated for about $30 \mathrm{~min}$ in ethanol. The zeta potential was measured in an electric field of -21.8 $\mathrm{V} / \mathrm{cm}$ with a current of $-0.02 \mathrm{~mA}$ and a $\mathrm{pH}$ of 7 . Three measurements were taken for each sample to provide an average reading.

X-ray diffraction (XRD) was shown the change of the MWNTs in the crystalline phase and lattice distortions. XRD data from the samples were collected using a Rigaku Model D/MAX diffractometer with $\mathrm{Cu} \mathrm{K} \alpha$ radiation. FT-Raman analyses were employed by using a Bruker Model RFS 100/S with the excitation wavelength of a He-Ne laser that was $600 \mathrm{~nm}$ and the power was $500 \mathrm{mV}$. The morphology of the acidtreated MWNTs was studied with transmission electron microscopy (TEM) measurement using a JEM 2100F, JEOL Co. at an electron microscope operating voltage of $200 \mathrm{kV}$. For the observations, samples were dispersed in ethanol in a low power sonication-bath for a few minutes and deposited on a holey carbon coated copper by drying a droplet of a suspension on the grid.

The hydrogen uptake experiment was performed using a BEL-HP from BEL-SORP Co. at $77 \mathrm{~K}$ and 1 atm. In each experiment, samples of about $0.5 \mathrm{~g}$ were loaded into a stainless chamber. Prior to the measurement, the system was evacuated for $6 \mathrm{~h}$ and the sample chamber was heated to $598 \mathrm{~K}$. Hydrogen was introduced after the chamber was cooled down to $77 \mathrm{~K}$ in liquid nitrogen. The hydrogen in the work was of an ultrahigh purity grade $(99.9999 \%)$ so that the influence of moisture and other impurities in the hydrogen source could be excluded. Finally, there was used a volumetric measurement to check the hydrogen uptake capacity.

\section{Results and Discussion}

Surface properties. Fig. 1 shows the FT-IR results of acidtreated MWNTs. The as-received MWNTs exhibit a less obvious FT-IR adsorption signature. The peaks between 800 and 1000 $\mathrm{cm}^{-1}$ in the spectrum are due to the stretching mode of aromatic amine groups, and the band between $1731 \mathrm{~cm}^{-1}$ and $1613 \mathrm{~cm}^{-1}$ corresponds to the stretching vibration mode of carbonyl $(\mathrm{C}=\mathrm{O})$, which may be introduced in the purification process and subsequent acid treatments. The peak at around $1700 \mathrm{~cm}^{-1}$, corresponds to the carboxyl stretching of the carboxyl groups, and the remarkable increase in that peak, during the acid-treatment confirms that the carboxyl groups have been successfully introduced on the surface of the MWNTs. ${ }^{11-13}$ This somewhat broad peak is attributed to other oxygen functional groups on the surfaces (such as lactone, anhydride, and quinine). The band between 2800 and $3000 \mathrm{~cm}^{-1}$ corresponds to the $\mathrm{C}-\mathrm{H}$ asymmetric and symmetric stretching vibration. This band shows that the acid treatment has an insignificant influence on the MWNTs. Note also that the $3480 \mathrm{~cm}^{-1}$ band, which corresponds to the stretching vibration of the hydroxyl groups, increases considerably after the acid treatment. Thus, the acid treatment makes the support surface rich in both weak and strong acid groups. The hydrophilic nature of the functional groups disperses the MWNTs. This result conforms to the results of other groups. ${ }^{14,16}$
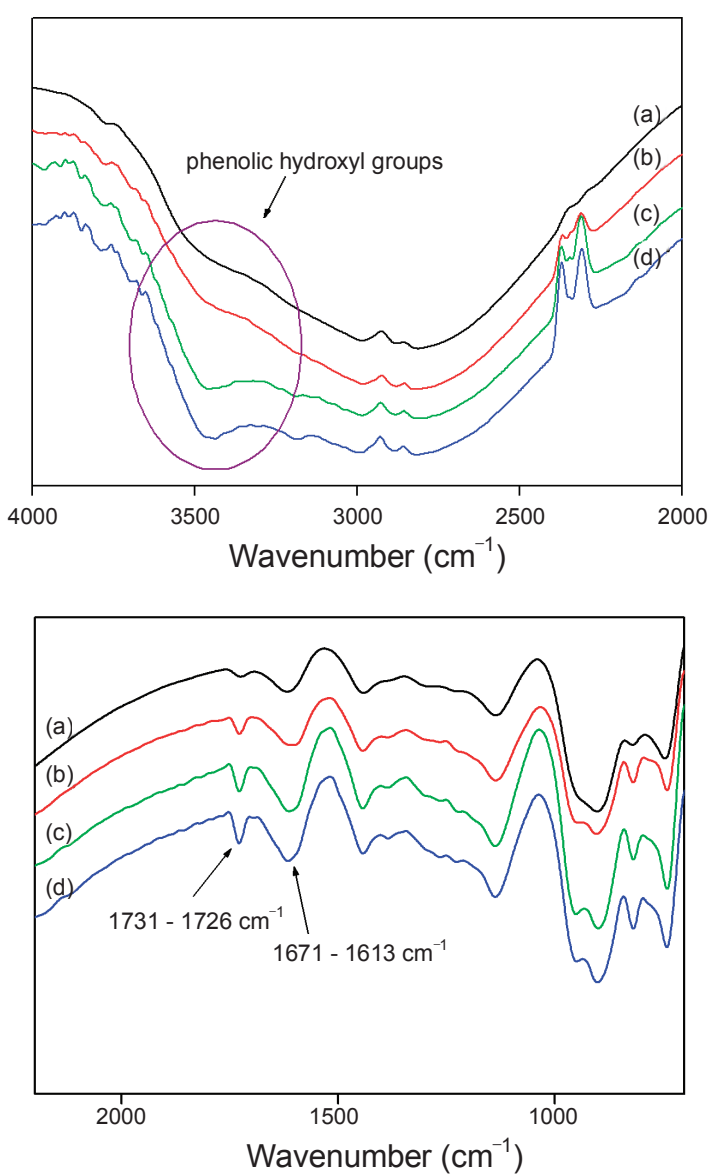

Figure 1. FT-IR spectra of acid-treated MWNTs; (a) as-received, (b) $\mathrm{T}-2$, (c) T-5, and (d) T-10.

Analysis of the XPS spectra helps determine the chemical species introduced by the acid treatment. The XPS spectra of the acid-treated MWNTs are shown in Fig. 2. The as-received MWNTs have few oxygen atoms due to the autoxidation in nature. In contrast, the acid-treated MWNT samples contain oxidized carbon atoms, such as carbonyl, carboxyl, and hydroxyl. ${ }^{15-17}$ An XPS peak fitting technique is used to confirm the distinction in the oxygen functional groups on the surface of the MWNTs. Table 1 lists the contents of the oxygen functional groups on the surface of MWNTs in terms of the area of each XPS subpeak fitting procedure. Clearly, the oxygen functional groups increase when the acid treatment is prolonged. Note especially that the growth of the hydroxyl groups is more prominent than that of the other groups. According to the literature, phenolic hydroxyl groups and carboxyl acid groups have a particularly strong acidity, whereas the carbonyl group has a weak or (neutral) acidity in terms of its acid-base characteristics. $^{18}$

Zeta potential. The acid-treated MWNTs were analyzed for surface charge by means of zeta-potential measurement. The zeta-potential ( $\zeta$ ) value was calculated using the Smoluchowski's equation (1), which can be expressed as:

$$
\zeta=\frac{4 \pi \eta U}{\varepsilon}
$$


(a)

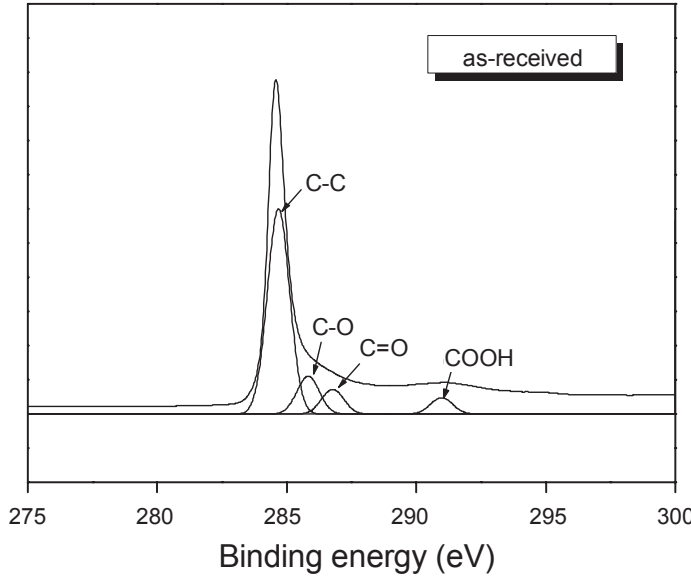

(b)

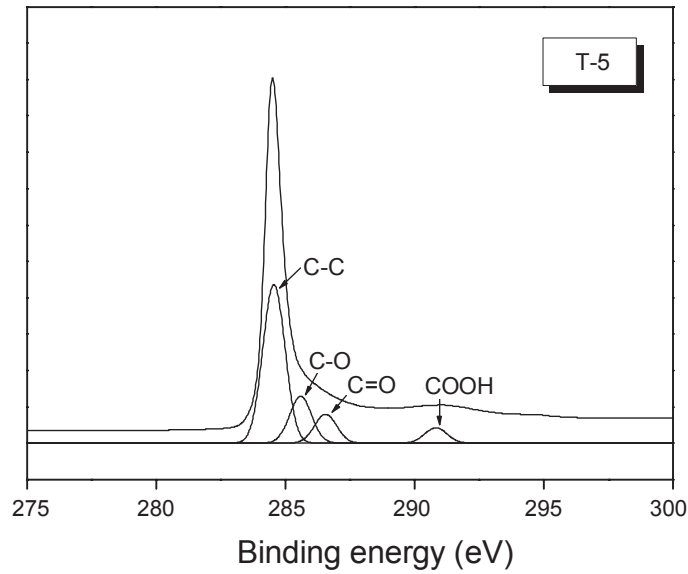

Figure 2. XPS $\mathrm{C}_{1 \mathrm{~S}}$ analysis spectra of as-received and T-5 samples.

Table 1. Compositions of acid-treated MWNTs obtained by XPS measurements

\begin{tabular}{ccccc}
\hline Specimens & $\mathrm{C}-\mathrm{C} \mathrm{( \% )}$ & $\mathrm{C}-\mathrm{OH}(\%)$ & $\mathrm{C}=\mathrm{O}(\%)$ & $\mathrm{COOH}(\%)$ \\
\hline as-received & 73.12 & 11.40 & 10.84 & 5.84 \\
$\mathrm{~T}-5$ & 70.21 & 13.98 & 10.50 & 5.65 \\
\hline
\end{tabular}

Table 2. Zeta-potential of acid-treated MWNTs

\begin{tabular}{cc}
\hline Specimens & Zeta-potential $(\mathrm{mV})$ \\
\hline as-received & -21.1 \\
$\mathrm{~T}-2$ & -33.4 \\
$\mathrm{~T}-5$ & -36.0 \\
$\mathrm{~T}-10$ & -41.1 \\
\hline
\end{tabular}

where $\eta$ is the coefficient of viscosity of the solvent, $U$ the degree of electrophoresis, and $\varepsilon$ the dielectric constant of the solvent.

The results of the zeta potential measurements are listed in Table 2 . The zeta potential absolute value increases gradually when the acid treatment is prolonged. The zeta potential absolute value of T-10 is the highest, namely $-41 \mathrm{mV}$; in contrast, the zeta potential of the as-received samples is the lowest, namely $-21 \mathrm{mV}$. This result can be attributed in part to the previously

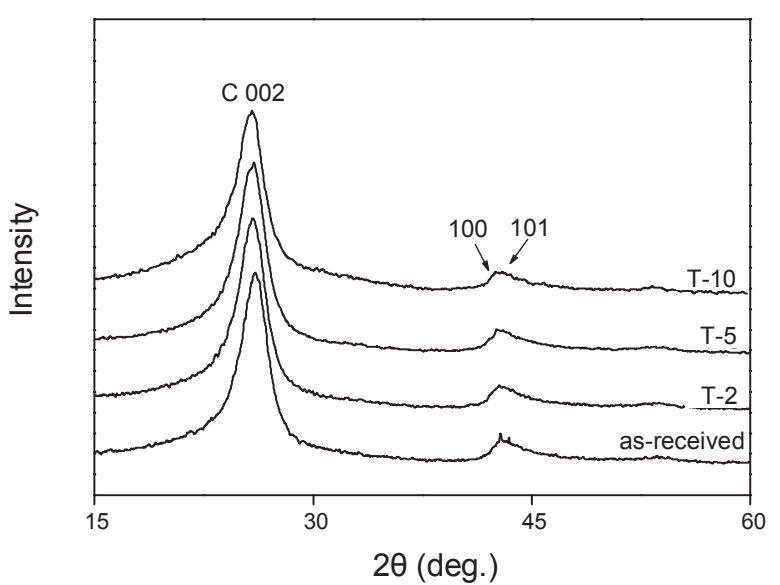

Figure 3. XRD spectra of acid-treated MWNTs.

Table 3. XRD parameters of acid-treated MWNTs

\begin{tabular}{ccc}
\hline Specimens & Angle $(\mathrm{C} \mathrm{002)}($ deg. $)$ & $(002) d$-spacing $(\AA)$ \\
\hline as-received & 26.04 & 3.422 \\
T-2 & 25.84 & 3.448 \\
T-5 & 25.82 & 3.451 \\
T-10 & 25.78 & 3.456 \\
\hline
\end{tabular}

mentioned factors of surface acidity and the dispersibility; furthermore, the introduction of acidic oxygen functional groups on the surface of the MWNTs can be expressed in terms of the zeta potential value. ${ }^{19}$

Structural properties. Fig. 3 shows the XRD spectra of acidtreated MWNTs. The XRD spectra are dominated by a peak at $2 \theta=26^{\circ}$, of the same intensity in acid-treated MWNTs, which is characteristic of highly structured graphitic carbon (002). Also, a weak peak and a shoulder were observed at $2 \theta=41.5$ and $44^{\circ}$, indicating (100) and (101) graphene layers, respectively. ${ }^{20}$ Table 3 lists the structural parameter of acid-treated MWNTs obtained by XRD analysis. The average $d$-spacing $(d)$ values calculated using Bragg's equation (2). It can be expressed as follows:

$$
n \lambda=2 d \sin \theta
$$

where $n$ is the number of order, $\lambda$ the wavelength of X-ray, $d$ the spacing between the planes in the atomic lattice, and $\theta$ the angle between the incident ray and the scattering planes.

The average $d$-spacing values lie in the range of 3.422 $3.456 \AA$. Therefore, we can note that acid-treatment does not have significantly influence on the average $d$-spacing, the crystalline phase, and the lattice distortions of the MWNTs. So, acid-treated MWNTs will possibly remain unchanged in respect of the crystallographic properties of MWNTs.

Raman spectroscopy is a very valuable tool for the characterization of carbon-based nanostructures. ${ }^{21-22}$ The Raman spectra of acid-treated MWNTs are shown in Fig. 4. All samples consist of two characteristic bands, namely the G-band at about 


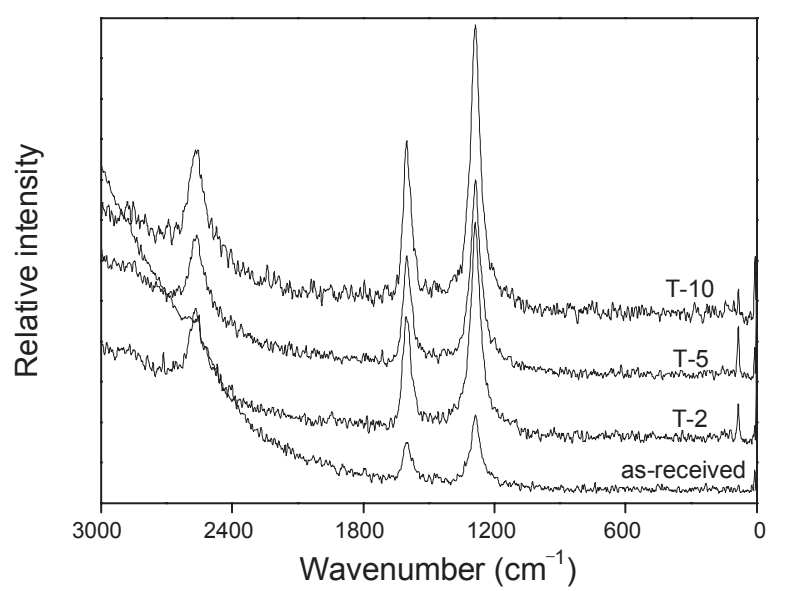

Figure 4. FT-Raman spectra of acid-treated MWNTs.
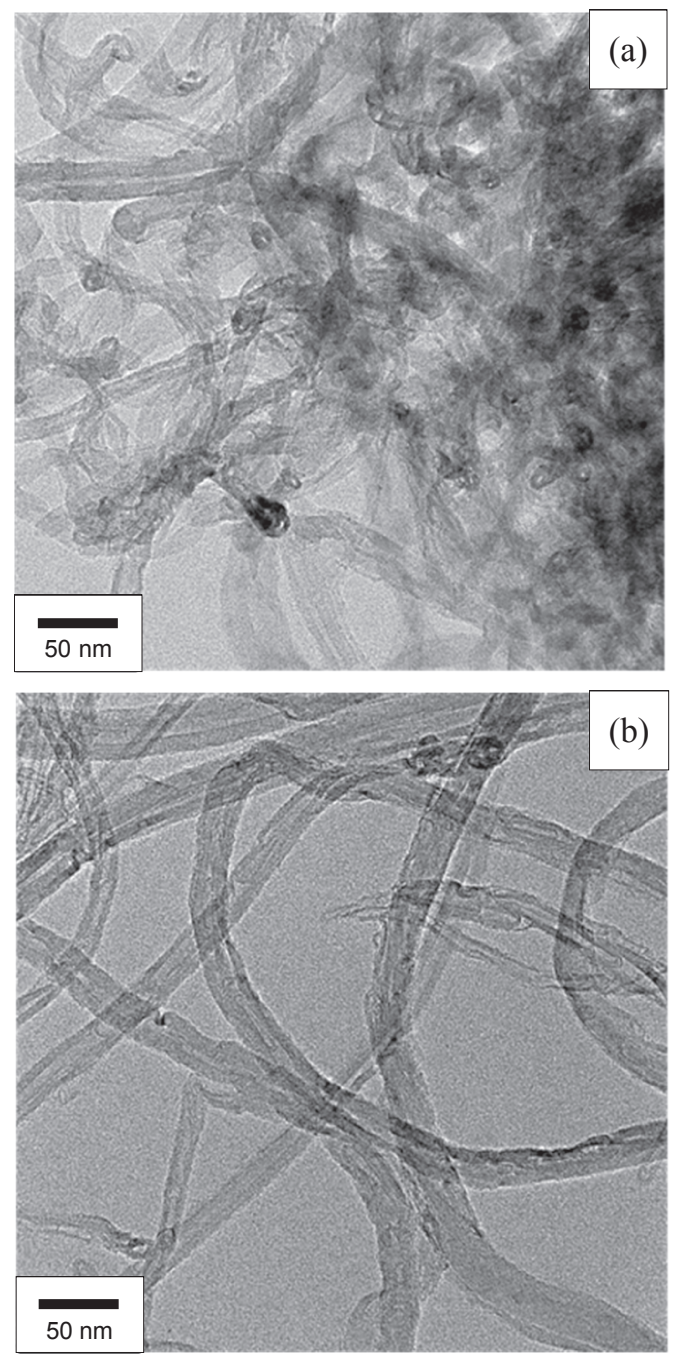

Figure 5. TEM images of as-received (a) and T-5 samples (b).

$1590 \mathrm{~cm}^{-1}$ and the D-band at about $1280 \mathrm{~cm}^{-1}$. The frequencies at around $1590 \mathrm{~cm}^{-1}$ were paid attention to, which corresponded to the tangential $\mathrm{C}-\mathrm{C}$ stretching vibration, known as a graphite mode G-band. At around $1280 \mathrm{~cm}^{-1}$, due to the local defects

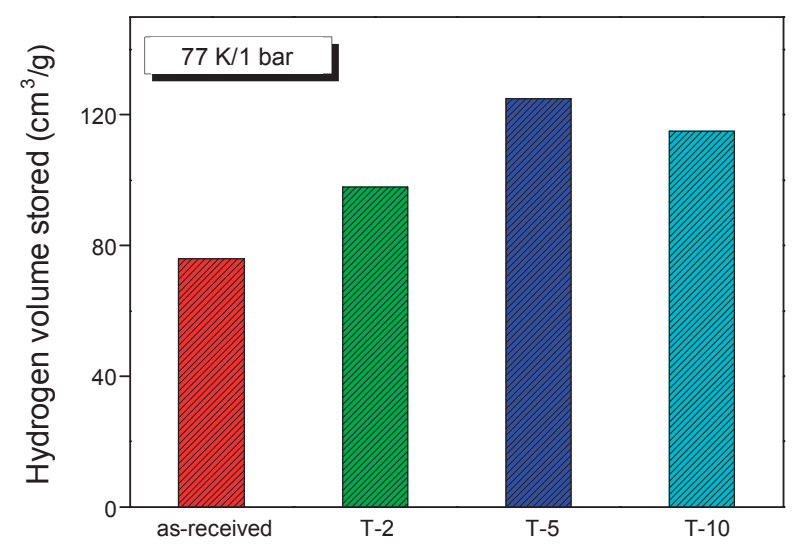

Figure 6. Hydrogen storage capacity of acid-treated MWNTs.

that originated from structural imperfections in the MWNTs, were named as defect mode D-band. The relatively strong Gband and weak D-band of all samples indicated that, when acidtreatments of MWNTs were performed, these characteristic peaks can still be identified, proving that the acid-treatments do not damage the structural ordering of MWNTs.

As shown in Fig. 5, TEM images used to confirm the morphologies of acid-treated MWNTs with high quality and high length/diameter ratio, which was also showed that the outer diameter was about 20 - $40 \mathrm{~nm}$. The acid-treated MWNTs do not reveal the erosion of their structures, such as the shortening and thinning of the MWNTs layers. So, it is showed that the morphologies of acid-treated MWNTs have significantly unchanged in comparison with as-received MWNTs.

Hydrogen storage capacity at low temperature. Fig. 6 illustrates the $\mathrm{H}_{2} / 77 \mathrm{~K}$ adsorption isotherms of acid-treated MWNTs. The experimental results confirm that the acid-treated MWNTs show greater improvement than the as-received MWNTs. MWNTs have a strong tendency to agglomerate in accordance with their nano-size and respective high surface energy. However, the grafting of oxygen functional groups, such as carboxylates, on the MWNTs imparts negative charges that create the electrostatic stability required for dispersion. ${ }^{23-24}$ The increase in the hydrogen storage capacity of acid-treated MWNTs is due to the oxygen functional groups on the surface of the MWNTs. The oxygen functional groups provide more hydrogen-related sites on the MWNTs. In particular, the portion of these groups having a predominantly acid character is charged to polarize the hydrogen molecules. Even though there is no charge transfer from the hydrogen to the oxygen functional groups, the chargeinduced dipole interaction produces the characteristic of adsorption. ${ }^{25-26}$ Thus, as shown in Table 2, there is a distinct increase in the hydrogen storage behaviors as the zeta potential absolute values increase, except for the T-10 sample. These results confirm that the acid treatment interferes with the hydrogen storage behaviors of the MWNTs.

\section{Conclusions}

The acid treatment produces an increase in the oxygen func- 
tional groups on the MWNTs when the treatment time is prolonged. The hydrogen storage capacity was enhanced in the presence of oxygen functional groups for samples T-2 and T-5. Therefore, the acid treatment can generate a higher amount of oxygen functional groups and these groups perform a significant role in terms of the dispersibility and enhanced hydrogen storage capacity of MWNTs. However, in the case of excessively treated MWNTs (T-10), the hydrogen storage capacity begins to decrease. Consequently, the optimization of MWNTs is essential for increasing the total hydrogen storage capacity.

Acknowledgments. This work was supported by the Materials Source Technology Development Program of the Ministry of Knowledge Economy, Korea.

\section{References}

1. Schlapbch, L.; Zuttel, A. Nature 2001, 414, 353.

2. Li, Y.; Zhao, D.; Wang, Y.; Xue, R.; Shen, Z.; Li, X. Int. J. Hydrogen Energy 2006, 32, 2513.

3. Peles, A.; van de Walle C, G. Phys. Rev. B 2007, 76, 214101.

4. Shindo, K.; Kondo, T.; Sakurai, Y. J. Alloy Compd. 2004, 372, 201.

5. Mu, S. C.; Tang, H. L.; Qian, S. H.; Pan, M.; Yuan, R. Z. Carbon 2006, 44,762 .

6. Endo, M.; Kroto, H. W. J. Phys. Chem. 1992, 96, 6941.

7. Saito, R.; Dresselhaus, G.; Dresselhaus, M. S. Chem. Phys. Lett. 1992, 195, 537

8. Chen, J.; Hamon, M. A.; Hu, H.; Chen, Y.; Rao, A. M.; Eklund, P. C.; Haddon, R. C. Science 1998, 282, 95.
9. Liu, J.; Rinzler, A. G.; Dai, H.; Hafner, J. H.; Bradley, R. K.; Boul, P. J.; Lu, A.; Iverson, T.; Shelimov, K.; Huffman, C. B.; RodriquezMacias, F.; Shon, Y. S.; Lee, T. R.; Colbert, D. T.; Smalley, R. E. Science 1998, 280, 1253.

10. Zhong, Z. Y.; Xiong, Z. T.; Sun, L. F.; Luo, J. Z.; Chen, P.; Wu, X.; Lin, H.; Tan, K. L. J. Phys. Chem. B 2002, 106, 9507.

11. Zhang, J.; Zou, H.; Qing, Q.; Yang, Y.; Li, Q.; Liu, Z.; Guo, X.; Du, Z. J. Phys. Chem. B 2003, 107, 3712.

12. Kim, Y. T.; Mitani, T. J. Power Sources 2006, 158, 1517.

13. Shen, J.; Huang, W.; Wu, L.; Hu, Y.; Ye, M. Mater. Sci. Eng. A 2007, 464, 151.

14. Kim, B. J.; Park, S. J. J. Colloid Interface Sci. 2007, 311, 619.

15. Cao, L.; Chen, H.; Wang, M.; Sun, J.; Zhang, X.; Kong, F. J. Phys. Chem. B 2002, 106, 8971.

16. Yu, H.; Jin, Y.; Peng, F.; Wang, H.; Yang, J. J. Phys. Chem. C 2008, 112,6758 .

17. Meng, H.; Sui, G. X.; Fang, P. F.; Yang, R. Polymer 2008, 49, 610.

18. Yue, Z. R.; Wang, W. J.; Gardner, S. D.; Pittman, C. U. Carbon 1999, 37, 1785.

19. Xu, R.; Wu, C.; Xu, H. Carbon 2007, 45, 2806.

20. Cuervo, M. R.; Esther, A. N.; Eva, D.; Ordonez, S.; Vega, A.; Ana, B. D.; Inmaculada, R. R. Carbon 2008, 46, 2096.

21. Shen, J. F.; Hu, Y. Z.; Qin, C.; Ye, M. X. Langmuir 2008, $24,3993$.

22. Liu, H.; Wang, X.; Fang, P.; Wang, S.; Qi, X.; Pan, C.; Xie, G.; Liew, K. M. Carbon 2010, 48, 721.

23. Zhao, N.; He, C.; Li, J.; Jiang, Z.; Li, Y. Mater. Res. Bull. 2006, 41, 2204.

24. Osorio, A. G.; Silveira, I. C. L.; Bueno, V. L.; Bergmann, C. P. Appl. Surf. Sci. 2008, 255, 2485.

25. Li, M.; Boggs, M.; Beebe, T. P.; Huang, C. P. Carbon 2008, 46, 466.

26. Leddy, L. M.; Ramaprabhu, S. Int. J. Hydrogen Energy 2007, 32 , 3998. 